\title{
GIANT HEMANGIOMA OF THE URINARY BLADDER: A CASE REPORT
}

\author{
MOHAMMAD GOLAM MOWLA CHOWDHURY, NASIMA AKTER, MD. SAJID HASSAN, ISTEAQ AHMED \\ SHAMEEM, MOHAMMAD SELIM MORSHED
}

Department of Urology, Bangabandhu Sheikh Mujib Medical University, Dhaka

\begin{abstract}
Case Summary:
We present a case of a 22 year-old male who had suffered from painless gross hematuria, occasional passage of clot and intermittent dysuria for last 3 years. On examination there were multiple reddish nodules all over the body including glens penis. USG showed extensive, solid, diffuse, soft tissue mass along the lateral and anterior walls of bladder. Cystoscopic examination revealed multiple bluish red papillary projection which occupying most of the anterior wall, both anterolateral and trigonal area of the urinary bladder. Resection biopsy was tried but failed to control extensive bleeding. Surgical treatment with total cystectomy and cutaneous urinary diversion was performed. Histopathology revealed hypertrophy smooth muscles with intervening vascular channels. The patient recovered smoothly with no complications encountered.
\end{abstract}

Bangladesh J. Urol. 2015; 18(1): 40-42

\section{Introduction}

Hemangiomas of the urinary bladder are rare benign tumors of the urinary bladder that account for $0.6 \%$ of all bladder tumors [1]. Initially they presented as bladder mass and finally cystoscopic biopsy and histopathology will diagnose the disease. They are mostly congenital in origin, arising from embryonic angioblastic stem cells [2]. At present there are approximately more than 100 cases reported in the literature, and treatment varies from transurethral resection to partial cystectomy and neodymium: yttrium aluminum garnet (ND-YAG) laser application. Herein, we present a case of a giant urinary bladder hemangioma that is failed to endoscope management but successfully treated with total cystectomy followed by cutaneous urinary diversion.

\section{Case Report}

A 22-year-old male patient visited to BSMMU outpatient clinic with the complaints of painless gross hematuria and intermittent dysuria last 3 years. Physical examination revealed no gross abnormality except multiple reddish nodules all over the body including glens penis. Initial work-up consisted of a complete blood count, coagulation and biochemical studies, urinalysis, ultrasonogram of the KUB region and cystoscopy.

Correspondences: Mohammad Golam Mowla Chowdhury, Email: uromowla@hotmail.com
Hematological and biochemical studies showed no abnormalities, and urinalysis revealed plenty of RBC per high-power field. Ultrasonogram showed extensive, solid, diffuse soft tissue mass along the lateral and anterior walls (Fig.1). Clinically he was diagnosed as bladder neoplasm. Cystoscopy revealed huge, diffuse lobulated growth bluish red in colour arising from bladder neck, base, lateral and anterior wall. Resection biopsy was tried but failed to control extensive bleeding by any means. Finally laparotomy was done with a lower midline incision. Angry looking dilated vessels were found in pelvis and around bladder neck. Finally total cystectomy and bilateral cutaneous ureterostomy was done.

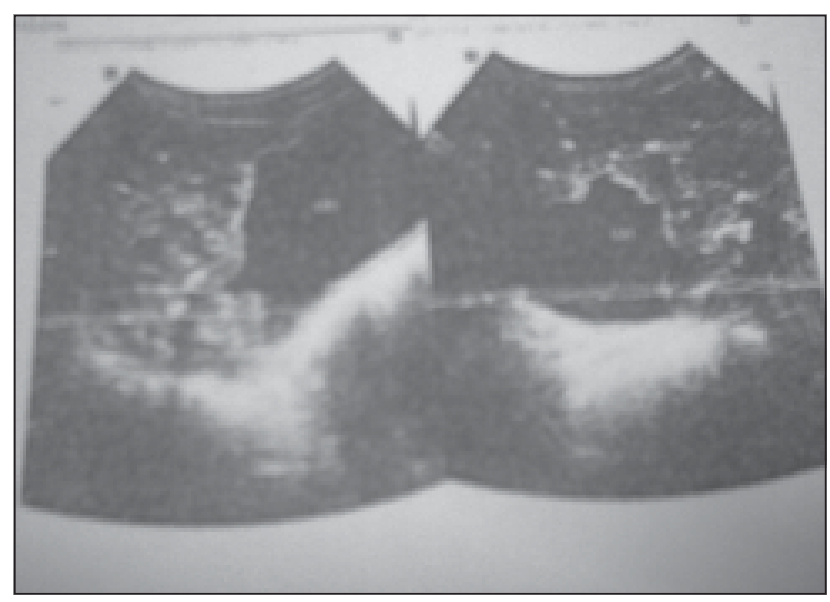

Fig.-1: USG of abdomen showing large bladder mass. 


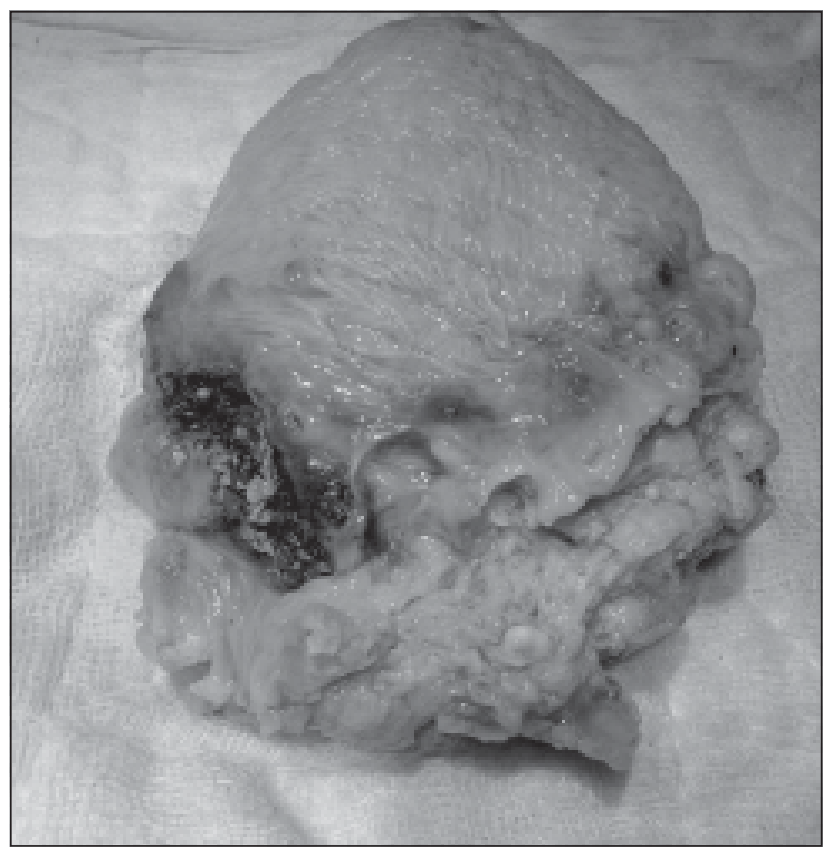

Fig.-2: Cystectomy specimen showing dilated blood vessels

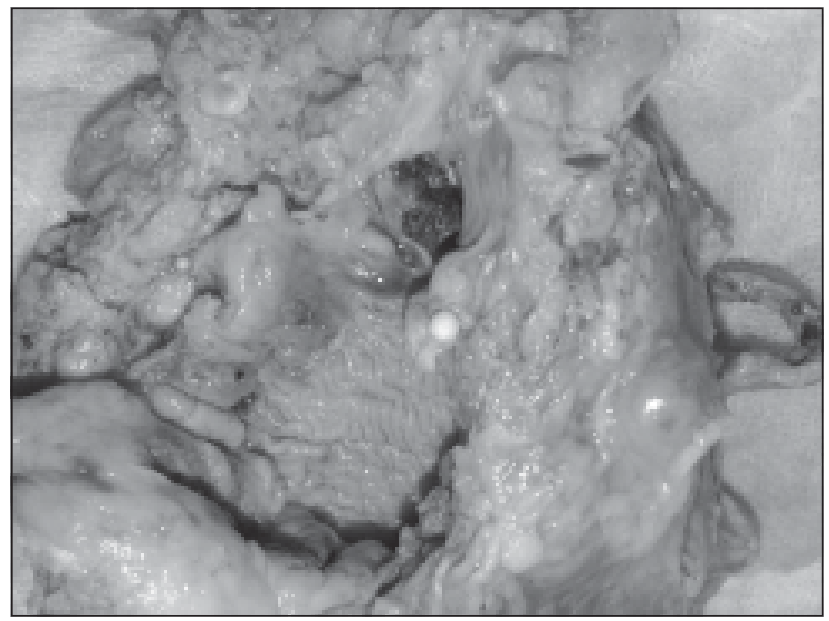

Fig.-3: Cystectomy specimen of everted bladder showing bluish nodules.

The bladder was explored, revealed a giant bluish-red vascular lesion occupying the anterior, both lateral walls including the neck of the urinary bladder (Figs. 2, 3). Penile nodule was excised. The specimens were for histological studies, and microscopic findings of urinary bladder revealed a picture of large vascular spaces filled with red blood cells and lined by flattened endothelium (Fig: 4).

Sections from penile nodule showed skin covered tissue and reveals multiple thin walled capillary channels. No granuloma or malignancy was seen (Fig. 4).

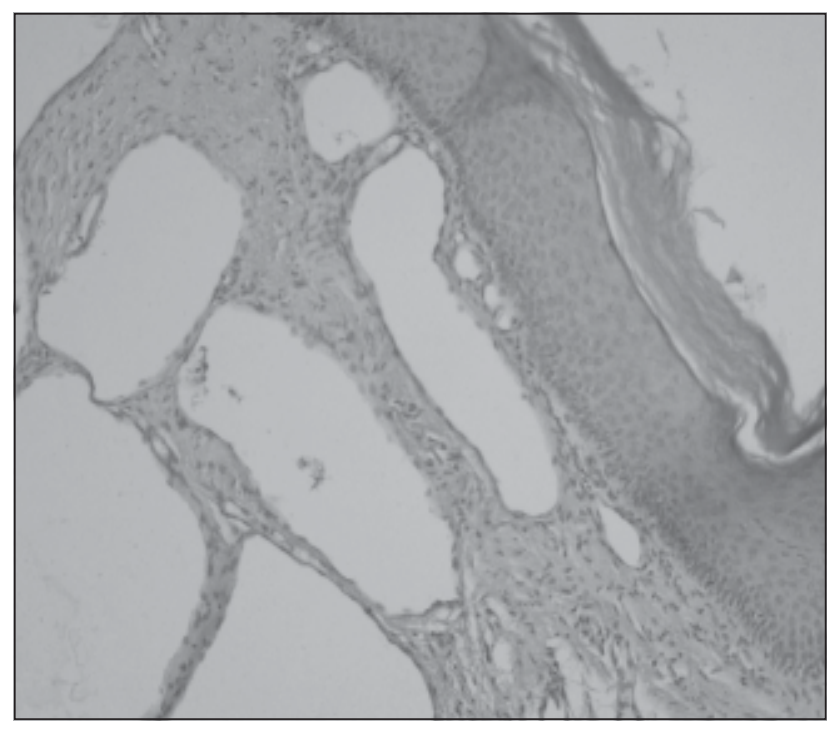

Fig.-4: Microscopic section of penile nodule shows dilated capillaries and skin.

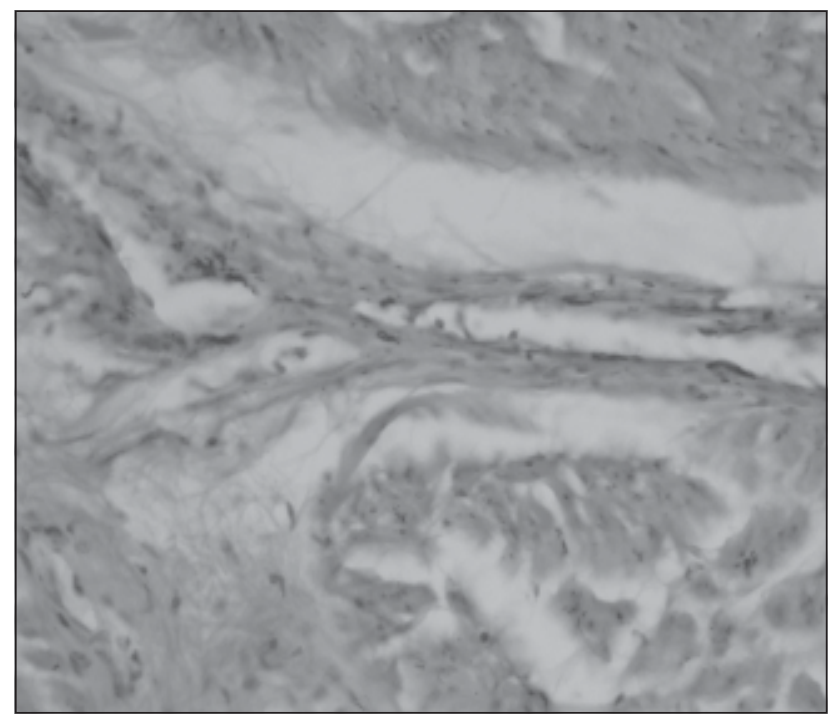

Fig.-5: Microscopic section of bladder smooth muscles and dilated vascular spaces.

\section{Discussion}

Hemangioma could occur in all the genitourinary system, urinary bladder is the second frequent place after the kidney [1]. It is a rare and benign mesechymal lesion. It represents only $0.6 \%$ of all bladder tumors [1-4]. More than 100 cases have been reported. Hemangiomas are classified as benign mesenchymal tumors, and as cavernous type, capilar type and arteriovenous type [5, 6]. Hemangiomas is not uncommon, but have been recognized in almost every organ of the body [7]. However, a primary hemangioma of the urinary bladder 
may occur singly or in association with a cutaneous or systemic vascular arteriovenous malformation [2]. A review of the literature in 1942 reported 40 cases of bladder hemangioma [5], and 6 more were reported by Melicow in 1955 among 954 cases of bladder tumors [1].

A bladder hemangioma may occur in any age group with no apparent gender predilection. It may coexist with a cutaneous hemangioma or be associated with Kippel- Trenaunay-Weber syndrome or Sturge-Weber syndrome with an estimated incidence of 3\% 6\% [8, 9]. The most common symptom is gross hematuria $[2,4,5,10]$, and other symptoms include irritative voiding symptoms and abdominal pain [5]. The majority of hemangiomas are solitary, small, and cavernous, with a predilection for the dome, posterior wall, and trigone of the bladder $[3,5]$. The clinical diagnosis is mainly established by cystoscopic, excretory urographic, ultrasound, CT-scan, and magnetic-resonance imaging (MRI) studies [5]. In the 1970's, pelvic angiography was used and was reported to be useful in establishing the preoperative diagnosis [7]. Hemangiomas appear as a sessile, broad-based, bluish-red mass on cystoscopy, with the excretory urogram, CT-scan, and MRI indicating the size and location of the hemangioma, which is a filling defect in $50 \%$ of cases $[3,7]$. The differential diagnosis with cystoscopy includes bladder vortices, endometrioma, sarcoma, malignant melanoma, and diffuse lymphangiohemangiomatosis [10]. An accurate diagnosis requires confirmation by biopsy. Histologically, a hemangioma is distinguished from an angiosarcoma and Kaposi's sarcoma by its lack of cytologic atypia [5]. However, some authors believe that when a hemangioma is suspected, a biopsy should be avoided because it may cause intractable bleeding, which can even be fatal $[4,7$, and 11$]$.

The management of bladder hemangiomas is controversial, and several therapeutic regimens have been proposed, all of which depend upon the extent of involvement. Therapeutic regimens include biopsy and fulguration, a partial or total cystectomy, irradiation, systemic steroids, sclerosing agent injection, interferon alpha- 2 therapies, and the recently more often used NGYAG laser therapy $[5,8]$. At present, a partial or total cystectomy is still performed for large hemangiomas or for hemangiomas with a high malignant potential according to the CT-scan and MRI findings [12] . Jason et al. reviewed 10 cases of angiosarcomas, in which 2 cases were related to a preexisting bladder hemangioma [12]. Consequently, a total cystectomy proved to be the better choice for our case, since the bladder hemangioma's were multiple large diffuse and occupied whole of the anterior wall, both lateral wall, trigon and bladder neck as identified by cystoscopy.

\section{References:}

1. Melicow MM. Tumors of the urinary bladder: A clinicopathological analysis of over 2500 specimens and biopsies. J Urol. 1955; 74: 498521.

2. Ikeda T, Shimamoto K, Tanji N, et al. Cavernous hemangioma of the urinary bladder in an 8-yearold child. Urol. Int. 2004; 11: 429-431.

3. Yasuhiro S, Hisashi K, Yoko K, et al. Hemangioma of the bladder with extravesical extension. Int. Urol. 1997; 59: 125-128.

4. Stanley KE Jr. Hemangioma-lymphangioma of the bladder in a child: report of a case with associated hemangiomas of the external genitalia. J. Urol. 1966; 96: 51-4.

5. Liang Cheng, Antonio G N, Roxann MN, et al. Hemangioma of the urinary bladder. Cancer 1999; 86: 498-504.

6. Rosenberg J, Golimbu M, Suares J et al. Congenital arteriovenous malformation of the bladder. J. Urol. 1973; 109: 605-608.

7. Fernandes $\mathrm{ET}^{1}$, Manivel JC, Reinberg Y. Hematuria in a new born infant caused by bladder hemangioma. Urology 1996; 47: 412-415.

8. Klein TW, Kaplan GW. Klippel-Trenaunay syndrome associated with urinary tract hemangioma. J Urol 1975; 114: 596-600.

9. Hendry WF, Vinnicombe J. Hemangioma of bladder in children and young adults. Br J Urol. 1971; 43: 309-316.

10. Proca $\mathrm{E}$. Hemangioma of the bladder. $\mathrm{Br} \mathrm{J}$ Urol 1977; 49:60.

11. Smith JA Jr. Lacer treatment of the bladder hemangioma. J Urol. 1990;143:282-294.

12. Engel JD, Kuzel TM, Moceanu MC, et al. Angiosarcoma of the bladder: a review. Urology 1998; 52: 778-784. 